\title{
Evaluation of 17 microsatellite markers for parentage testing and individual identification of domestic yak (Bos grunniens)
}

\author{
Jie Pei ${ }^{1,2}$, Pengjia Bao ${ }^{1,2}$, Min Chu ${ }^{1,2}$, Chunnian Liang ${ }^{1,2}$, Xuezhi Ding ${ }^{1,2}$, Hongbo Wang ${ }^{1,2}$, Xiaoyun Wu ${ }^{1,2}$

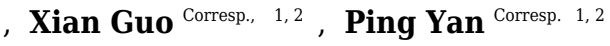 \\ ${ }^{1}$ Animal Scienece Department, Lanzhou Institute of Husbandry and Pharmaceutical Sciences, Chinese Academy of Agricultural Sciences, Lanzhou, Gansu, \\ China \\ ${ }^{2}$ Key Laboratory for Yak Genetics, Breeding, and Reproduction Engineering of Gansu Province, Lanzhou, Gansu, China \\ Corresponding Authors: Xian Guo, Ping Yan \\ Email address: guoxian@caas.cn, pingyan63@126.com
}

Background. Yak (Bos grunniens) is the most important domestic animal for people living at high altitudes. Yak ordinarily feeds by grazing, and this behavior impacts the accuracy of the pedigree record because it is difficult to control mating in grazing yak. This study aimed to evaluate the pedigree system and individual identification in polled yak.

Methods. Seventy-one microsatellite loci were selected from the literature, mostly from the studies on cattle. Thirty-five microsatellite loci generated excellent PCR results and were evaluated for the parentage testing and individual identification of 236 unrelated polled yaks. Seventeen of these 35 microsatellite loci had polymorphic information content (PIC) values greater than 0.5 , and these loci were in Hardy-Weinberg equilibrium without linkage disequilibrium.

Results. Using multiplex PCR, capillary electrophoresis, and genotyping, very high exclusion probabilities were obtained for the combined core set of 17 loci. The exclusion probability (PE) for one candidate parent when the genotype of the other parent is not known was 0.99718116. PE for one candidate parent when the genotype of the other parent is known was 0.99997381 . PE for a known candidate parent pair was 0.99999998 . The combined PEI (exclusion probability for identity of two unrelated individuals) and PESI (exclusion probability for identity of two siblings) were $>0.99999999$ and 0.99999899 , respectively. These findings indicated that the combination of 17 microsatellite markers could be useful for efficient and reliable parentage testing and individual identification in polled yak.

Discussion. Many microsatellite loci have been investigated for cattle paternity testing. Nevertheless, these loci cannot be directly applied to yak identification because the two bovid species have different genomic sequences and organization. Seventeen loci were selected from 71 microsatellite loci based on efficient amplification, unambiguous genotyping, and high PIC values for polled yaks, and were suitable for parentage analysis in polled yak populations. 
1 Evaluation of $\mathbf{1 7}$ microsatellite markers for parentage testing and individual identification

2 of domestic yak (Bos grunniens)

3

4 Jie Pei ${ }^{1,2}$, Pengjia Bao ${ }^{1,2}$, Min Chu ${ }^{1,2}$, Chunnian Liang ${ }^{1,2}$, Xuezhi Ding ${ }^{1,2}$, Hongbo Wang ${ }^{1,2}$,

5 Xiaoyun $\mathrm{Wu}^{1,2}$, Xian Guo ${ }^{1,2}$ and Ping Yan ${ }^{1,2}$

6

$7 \quad{ }^{1}$ Lanzhou Institute of Husbandry and Pharmaceutical Sciences, Chinese Academy of

8 Agricultural Sciences, Lanzhou, China

$9{ }^{2}$ Key Laboratory for Yak Genetics, Breeding, and Reproduction Engineering of Gansu Province,

10 Lanzhou, China

11

12 Corresponding authors:

13 Xian Guo,guoxianlz@163.com

14 Ping Yan, pingyan63@126.com

15 
16

17

\section{ABSTRACT}

Background. Yak (Bos grunniens) is the most important domestic animal for people living at high altitudes. Yak ordinarily feeds by grazing, and this behavior impacts the accuracy of the pedigree record because it is difficult to control mating in grazing yak. This study aimed to evaluate the pedigree system and individual identification in polled yak.

Methods. Seventy-one microsatellite loci were selected from the literature, mostly from the studies on cattle. Thirty-five microsatellite loci generated excellent PCR results and were evaluated for the parentage testing and individual identification of 236 unrelated polled yaks. Seventeen of these 35 microsatellite loci had polymorphic information content (PIC) values greater than 0.5, and these loci were in Hardy-Weinberg equilibrium without linkage disequilibrium.

Results. Using multiplex PCR, capillary electrophoresis, and genotyping, very high exclusion probabilities were obtained for the combined core set of 17 loci. The exclusion probability (PE) for one candidate parent when the genotype of the other parent is not known was 0.99718116 . PE for one candidate parent when the genotype of the other parent is known was 0.99997381. PE for a known candidate parent pair was 0.99999998. The combined PEI (exclusion probability for identity of two unrelated individuals) and PESI (exclusion probability for identity of two siblings) were $>0.99999999$ and 0.99999899 , respectively. These findings indicated that the combination of 17 microsatellite markers could be useful for efficient and reliable parentage testing and individual identification in polled yak.

Discussion. Many microsatellite loci have been investigated for cattle paternity testing. Nevertheless, these loci cannot be directly applied to yak identification because the two bovid species have different genomic sequences and organization. Seventeen loci were selected from 71 microsatellite loci based on efficient amplification, unambiguous genotyping, and high PIC values for polled yaks, and were suitable for parentage analysis in polled yak populations. 


\section{INTRODUCTION}

43

Yak (Bos grunniens), a member of Bovidae family, has successfully been adapted to the severe cold and low oxygen levels, characteristics of high altitude regions $(\sim 2,500-5,500 \mathrm{~m})$, such as the Himalayas in South-Central Asia, the Qinghai-Tibetan Plateau, Mongolia, and Russia (Wu 2016). These regions are known for their high elevations, pristine natural environments, and extreme seasonal variations (Mizuno et al. 2015). The ability of yak to survive in such rugged natural environment is due to its varied behavioral, physiological, and genetic adaptations (Barsila et al. 2014; Hu et al. 2012; Qiu et al. 2012). For instance, remarkable reduction of heat production at night when not grazing and increased energy consumption when grazing under free-range conditions enables yaks to save more energy and resist the extremely harsh conditions than other cattle under similar environmental conditions (Ding et al. 2014). Yak can thrive under extreme environmental conditions, such as the Tibetan Plateau where few other animals can survive. In this region, yak have significantly contributed to human life by providing meat, milk, fur, leather, and transportation compared to other animals (Medhammar et al. 2012; Wang et al. 2018).

There are about 13 million domestic yak in China, accounting for approximately $90 \%$ of the global yak population. Although there are 18 yak breeds in China, only one breed (Datong) has been included in a breeding program ( $\mathrm{Wu} 2016)$. However, polled yak has been bred for many years at the foot of Ashidan Mountain, as polling reduces the risk of horn-inflicted injury or death among the herdsmen. Accurate genealogical records can help estimate genetic parameters and improve breeding programs, ensuring efficient and effective breeding progression to avoid excessive inbreeding. However, it is difficult to control mating among yaks while grazing. Furthermore, semen samples could be erroneously mislabeled during preparation, and mating records might be misinterpreted because of clerical errors made during artificial insemination. Therefore, accurate yak pedigree records that are compiled by parentage testing and individual identification are essential to the yak breeding process. 
69

70

parentage verification and individual identification (Estoup et al. 2002). Microsatellites refer to short tandem repeats (STRs) or simple sequence repeats (SSRs), and are considered as tracts of DNA motifs ranging from one to 10 nucleotides in length with repeats of 5-50 times (Carneiro Vieira et al. 2016). Microsatellites can be used to develop pedigree animal populations and evaluate animal breeding, supporting genetic improvement by selective breeding (Weising et al. 1998). The application of microsatellites as molecular markers for animal identification and parentage verification produced highly accurate and effective results in both breeding and forensics (Linacre et al. 2011).

Microsatellite marker analysis has been used to verify the parentage in breeding registries and identify individual animals that are linked to a particular database or owner. Microsatellite panels of cattle (Zhao et al. 2017), horse (Kang et al. 2016), sheep (Rosa et al. 2013), dog (Jeong et al. 2015), and parrot (Coetzer et al. 2017) have been well characterized. Parentage control in the beef cattle breeds, Charolais, Limousin, and Preta, in Portugal was assessed using 10 microsatellite markers, and the results revealed a combined exclusion probability (PE) above 0.9995, indicating their ability to exclude a random parent pair (Carolino et al. 2009). The application of 11 microsatellite loci in paternity testing in Yugoslav Pied cattle breed in Serbia revealed a combined PE of 0.999 (Stevanovic et al. 2010). Sixteen specific microsatellite markers were used to develop a genetic system of meat traceability for several beef cattle breeds, including Japanese Black, Anduo yak, Limousin, Jiaxian Red, Nanyang Yellow, and Luxi Yellow (Zhao et al. 2017). Previous studies reported that microsatellite genotyping was used for population genetics analysis and parentage testing in yak. However, these loci and their primers were originally developed for cattle, and then used directly in yak (Li et al. 2013; Nguyen et al. 2005). Therefore, there is a need for explorative and application-based studies on microsatellite markers or panels of microsatellite markers that are suitable for accurate individual identification and parentage testing in yak.

Hence, in the present study, we aimed to establish a paternity test and individual identification system for the polled yak. Therefore, the study was expected to (1) calculate the genetic 
96

97

parameters of polled yak microsatellite loci, which have been commonly reported by population genetics studies of cattle and yak; (2) evaluate the application values of the loci with high polymorphic information content (PIC) for parentage testing and individual identification; and (3) explore a multi-loci combination test system for parentage testing and individual identification.

\section{MATERIALS AND METHODS}

\section{Marker selection and primer design}

The microsatellites used in the present study were selected from the previous reports on cattle breeding based on the following criteria: (a) high PIC; (b) large number of alleles; (c) relatively infrequent null alleles; and (d) homogeneous or approximately homogeneous repeat motifs (Schnabel et al. 2000). Among the 71 bovine microsatellite markers selected, 65 were derived from cattle, and 6 were exclusive to yak (Supplemental Table S1). All 71 microsatellites and their flanking sequences were found in the cattle genome, and were searched for in the yak genome. The primers for most loci used in previous studies were not suitable for the yak genome because of low scores. However, the primers used to amplify BM1824, BM2113, BMS2533, ETH121, ETH225, ILSTS008, INRA124, RM099, INRA126, UMN0103, UMN0307, UMN0920, UMN2303, UMN3007, and UMN3008 loci presented relatively high scores, so these loci were not redesigned for yak. The primers used in the present study are listed in Table S1.

\section{Sample collection}

Polled yaks bred via selective breeding were selected from herds in Ashidan Mountain region in Qinghai Province, China. To avoid consanguinity, samples were taken from animals with no genetic relationship. All yaks were handled in strict accordance with good animal practices by following the Animal Ethics Procedures and Guidelines of the People's Republic of China. The 
121 present study was approved by the Animal Administration and Ethics Committee of Lanzhou

122

123

124

125

126

127

128

129

130

131

132

133

134

135

136

137

138

139

140

141

142

143

144

145

146

Institute of Husbandry and Pharmaceutical Sciences of Chinese Academy of Agricultural

Sciences (Permit No. SYXK-2016-0039). Blood was drawn from the jugular veins of 236

unrelated individuals, including 38 sires and 198 dams, and samples were mixed with

preservation buffer (containing $1.5 \mathrm{mg} \mathrm{mL}^{-1}$ EDTA and $137 \mathrm{mmol} \mathrm{L}^{-1} \mathrm{NaCl}$ ) at a ratio of 5:1. The blood samples were stored at $-80{ }^{\circ} \mathrm{C}$ in an ultra-cold freezer until DNA extraction.

\section{DNA extraction and quantification}

Genomic DNA was extracted from white blood cells, separated from whole blood, and digested with proteinase $\mathrm{K}$. After digestion, the samples were centrifuged at $5000 \times g$ for $2 \mathrm{~min}$, and the resulting supernatant (clear aqueous layer) was transferred to a new test tube. After the addition of $0.5 \mathrm{~mL}$ of $10 \mathrm{mg} \mathrm{mL}^{-1}$ RNase A, DNA was individually extracted with a phenol:chloroform:isoamyl alcohol mixture (25:24:1) followed by chloroform, precipitated with ethanol, and resuspended in $50 \mathrm{~mL}$ TE buffer (10 mM Tris-HCl and $1 \mathrm{mM}$ EDTA, $\mathrm{pH}$ 8.0). The extracted DNA was then quantified using a NanoDrop 2000 fluorometer (Thermo Fisher Scientific, Waltham, MA, USA).

\section{Preliminary primer screening}

Unlabeled primer pairs (Table S1) for each microsatellite marker were used for amplification of DNA fragments. PCR was performed with a reaction mixture at a total volume of $20 \mu \mathrm{L}$, comprising of 20-50 ng genomic DNA, $10 \mathrm{mM}$ Tris- $\mathrm{HCl}$ (pH 9.0), $50 \mathrm{mM} \mathrm{KCl,} 1.5 \mathrm{mM} \mathrm{MgCl}_{2}$, $2.0 \mathrm{mM}$ each dNTP, $5 \mathrm{ng}$ of bovine serum albumin, and $1.0 \mathrm{U}$ of $\mathrm{Taq}^{\mathrm{TM}}$ Hot Start Version polymerase (TaKaRa Bio Inc., Kusatsu, Shiga, Japan). Primer concentrations ranged from 1.0 to $5.5 \mu \mathrm{M}$. The thermal cycle parameters were as follows: 5 min at $95^{\circ} \mathrm{C} ; 35$ cycles of $30 \mathrm{~s}$ at 95 ${ }^{\circ} \mathrm{C}, 30 \mathrm{~s}$ at $55-58{ }^{\circ} \mathrm{C}$ (Table S1), and $20 \mathrm{~s}$ at $72{ }^{\circ} \mathrm{C}$; and a final extension step for $5 \mathrm{~min}$ at $72{ }^{\circ} \mathrm{C}$. Amplifications were performed on a Veriti 96-Well Thermal Cycler (Applied Biosystems Corp., 
147 Foster City, CA, USA). The amplicons were visualized on 2\% agarose gels (Gene Tech Co. Ltd., 148 Chai Wan, Hong Kong, PRC). Five microliters of PCR product were mixed with $1 \mu \mathrm{L}$ of loading 149 dye (TaKaRa Bio Inc.) and electrophoresed for $35 \mathrm{~min}$ at $85 \mathrm{~V}$. The samples were then 150 visualized using the GelDoc ${ }^{\mathrm{TM}} \mathrm{XR}+$ gel imaging system (Bio-Rad Corp., Hercules, CA, USA).

151 The amplicons with high specificity and high amplification efficiency, whose loci were either

152 non-syntenic or separated by $>10 \mathrm{cM}$ (to avoid strong genetic linkage) (Table S2), were manually cut out of agarose gels and forwarded to Invitrogen (Carlsbad, CA, USA) and Thermo Fisher Scientific without prior purification for Sanger sequencing on an ABI3730xl automated sequencer (Applied Biosystems Corp.) to identify repeat markers. Only the forward primers were used to sequence the target markers.

\section{Genetic information acquisition}

The confirmed primer sequences flanking the microsatellite loci were synthesized with a fluorescent label $\left(\mathrm{FAM}^{\mathrm{TM}}, \mathrm{HEX}^{\mathrm{TM}}\right.$, or TAMRA ${ }^{\mathrm{TM}}$; Thermo Fisher Scientific) attached to the 5' end of each forward primer. The microsatellites were separately amplified by PCR to identify the highly polymorphic loci. The amplification systems and conditions were similar to those described above for unlabeled primers.

After amplification, $1 \mu \mathrm{L}$ of amplified fragment mix was added to $0.5 \mu \mathrm{L}$ of loading buffer (blue dextran, $50 \mathrm{mg} \mathrm{mL}^{-1}$; EDTA, $25 \mathrm{mM}$ ) and $4 \mu \mathrm{L}$ of deionized formamide, and it was then denatured by incubation for $5 \mathrm{~min}$ at $95^{\circ} \mathrm{C}$. An internal size standard $(0.5 \mu \mathrm{L}$ of Thermo ABI 4322682; Thermo Fisher Scientific) was added to each sample, and the fluorescently labeled PCR products were then separated by capillary electrophoresis (ABI3730xl Genetic Analyzer; Applied Biosystems Corp.). The fluorescently labeled DNA fragments were first analyzed with GENESCAN v. 3.7 (Applied Biosystems Corp.) followed by GENOTYPER v. 3.7 NT (Applied Biosystems Corp.). The fragments were then automatically sorted according to the internal size standard. The numbers of microsatellite repeat motifs were calculated based on the amplicon lengths (Table S3). 
175

176

177

178

179

180

181

182

183

184

185

186

187

188

189

190

191

192

193

194

195

196

197

198

\section{Genetic information analysis}

The genotypic data were initially processed in Microsoft Office Excel 2007, manually checked for errors, and then transformed into input files that were required for subsequent analyses. The performance characteristics [observed heterozygosity (HO), expected heterozygosity (HE), PIC, estimated null allele frequency (F(null)), Hardy-Weinberg equilibrium (HWE), and linkage disequilibrium] were measured with GENEPOP v. 4.6 (Raymond \& Rousset 1995; Rousset 2008) and CERVUS v. 3.0.7 (Kalinowski et al. 2010; Slate et al. 2000).

\section{Multiplex PCR conditions}

Genotyping of 236 yaks for 35 loci (Table 1) produced a core set of 17 loci with high PIC values (Table 2). Four multiplex PCR reactions were assembled, each containing four or five microsatellite markers. The primer sequences and concentrations used in the multiplex PCR reactions are shown in Table 2. The multiplex PCR reactions were performed with reaction mixtures at a total volume of $15 \mu \mathrm{L}$, containing $25 \mathrm{ng}$ of genomic DNA, $10 \mathrm{mM}$ Tris- $\mathrm{HCl}(\mathrm{pH}$ 8.3), $35 \mathrm{mM} \mathrm{KCl}, 1.8 \mathrm{mM} \mathrm{MgCl}_{2}, 5.0 \mathrm{mM}$ each dNTPs, and $2.5 \mathrm{U}$ of $\operatorname{Taq}^{\mathrm{TM}}$ Hot Start Version polymerase (TaKaRa Bio Inc.). Amplifications were performed in a Veriti 96-Well thermal cycler (Applied Biosystems Corp.) under the following conditions: $95{ }^{\circ} \mathrm{C}$ for $5 \mathrm{~min}$; 25 cycles of $95{ }^{\circ} \mathrm{C}$ for $30 \mathrm{~s}, 55-58{ }^{\circ} \mathrm{C}$ for $30 \mathrm{~s}$, and $72{ }^{\circ} \mathrm{C}$ for $30 \mathrm{~s}$; 10 cycles of $95{ }^{\circ} \mathrm{C}$ for $30 \mathrm{~s}, 53{ }^{\circ} \mathrm{C}$ for 30 $\mathrm{s}$, and $72{ }^{\circ} \mathrm{C}$ for $30 \mathrm{~s}$; and final extension at $72{ }^{\circ} \mathrm{C}$ for $5 \mathrm{~min}$. Genotyping of these microsatellite loci was performed as described above.

\section{Parentage testing and individual identification}

Exclusion probability values for parentage testing were obtained using a likelihood-based method based on genotypic information. Five types of PEs were calculated for the set of loci in 
199

200

201

202

203

204

205

206

207

208

209

210

211

212

213

214

215

216

217

218

219

220

221

222

223

224

CERVUS v. 3.0.7. PE1 was defined as the average probability of excluding an unrelated candidate parent of an arbitrary offspring when the genotype of the other parent is unknown. PE2 was defined as the average probability of excluding an unrelated candidate parent of an arbitrary offspring when the genotype of the other parent is known. PEP was defined as the average probability of excluding a pair of unrelated candidate parents of an arbitrary offspring. PEI was defined as the average probability of differentiating two randomly-selected individuals. PESI was defined as the average probability of differentiating two randomly-selected full siblings (Kalinowski et al. 2010; Slate et al. 2000).

\section{RESULTS}

\section{Microsatellite loci characteristics}

After preliminary screening, a total of 35 microsatellite loci with the highest primer specificity among the 71 microsatellite loci were selected for further analysis. The number of alleles, allele size range, HO, HE, PIC, F(null), and HWE of the 35 microsatellite loci of polled yaks are presented in Table 1. Three monomorphic loci, namely BM2943, INRA035, and RM099, were identified. The remaining 32 loci were polymorphic. The number of alleles per locus ranged from three (CSSM013 and CSSM033) to 12 (SPS115), and PIC values ranged from 0.084 (MM12) to 0.815 (ILSTS028). Four of these 32 polymorphic loci had low PIC values (i.e., < 0.25), 11 had moderate PIC values (0.25-0.50), and 17 loci had high PIC values $(>0.50)$ (Table $1)$.

The 17 genetic markers with high PIC values were reproducible, informative, and locusspecific for parentage testing and individual identification. The number of alleles ranged from five (BM720, INRA005, TGLA227) to 12 (SPS115). Heterozygosity values ranged from 0.573 (POTCHA) to 0.806 (ILSTS028), with an average of 0.662. Deviations between HO and HE ranged from 0.003 (INRA005) to 0.103 (TGLA126). The 17 loci had an average PIC values of 0.636, ranging from 0.529 (INRA005) to 0.815 (ILSTS028). The null allele frequency ranged 
225

226

227

228

229

230

231

232

233

234

235

236

237

238

239

240

241

242

243

244

245

246

247

248

249

from 0.0015 (TGLA227) to 0.0819 (TGLA126) (Table 1).

\section{Hardy-Weinberg and linkage disequilibrium tests}

The results of HWE tests of the 17 microsatellite loci indicated no significant differences ( $\mathrm{P}>$ $0.05)$ (Table 1). Therefore, the 17 loci with high PIC values $(>0.5)$ were selected for yak paternity testing. Linkage disequilibrium within polled yaks resulted in 136 comparisons, and no interlocus disequilibrium was detected between loci on the same chromosome (Table S6).

\section{Multiplex amplification and loading}

Multiplex PCR reactions were organized to co-amplify four groups of 4-5 loci with high PIC values. Non-overlapping allele lengths that presented the same fluorescent color label were selected. Typical fluorescence signals of the core 17 microsatellite loci are shown in Figure 1. Using the three available fluorescent colors, we multiplex-loaded and scored two groups of eight or nine loci in each run.

\section{Parentage inference}

The exclusion probabilities were calculated from the allele frequencies based on the underlying assumptions of HWE. The PE1 values of the 17 core loci ranged from 0.189 (BM720) to 0.503 (ILSTS028), with an average of 0.285. The average PE2 values of the 17 markers was 0.451 , and values ranged from 0.328 (INRA005) to 0.673 (ILSTS028). Regarding the 17 core loci, the combined PE1 and PE2 values were 0.99718116 and 0.99997381 , respectively. For the putative parents, the combined PEP value was 0.99999998. Combined PEI and PESI values were > 0.99999999 and 0.99999899 , respectively (Table 2). Therefore, the PE values indicated that the discriminatory power of the 17 loci was high. 


\section{DISCUSSION}

251 Previous studies indicated that $4.3 \%$ of annual losses with regard to genetic gain during dairy

252 breeding were caused by pedigree errors (10\%), compared to simulation analysis of accurate

253 paternity determination data(Israel \& Weller 2000). In fact, the pedigree error rate in yaks was

254 high due to incorrect paternity as yaks feed primarily by grazing, thwarting parentage attribution.

255 Additionally, clerical and insemination errors and fading ink on artificial insemination records

256 and labels might contribute to sample mixing, thereby, leading to pedigree errors. Therefore, it is

257 necessary to identify and correct the pedigree through parentage testing and individual

258 identification. These practices that aim at genetic improvement of yak are essential for

259

260

261

262

263

264

265

266

267

268

269

270

271

272

273

274

275

276 generating reliable breeding programs. Several reports have been published on the use of microsatellite markers for cattle identification (Sharma et al. 2015; Zhao et al. 2017), but the performance characteristics of yak identification panels have not yet been established. Parentage testing for yak breeding increases the profitability by improving the efficiency of selective breeding programs.

The most commonly used methods of livestock identification and parentage verification rely on microsatellites (Jan \& Fumagalli 2016; Jeong et al. 2015; Wang et al. 2017). However, single nucleotide polymorphisms (SNPs) have been applied in the identification and parentage verification of swine (Sus scrofa) and cattle (Bos taurus) (Eggen 2012; Rohrer et al. 2007). A recent study debated on the use of SNPs instead of microsatellites for parentage verification (Kaiser et al. 2017). However, at least 200 SNPs should be used for parentage testing to reduce false-negative results, and at least 700 SNPs are required to completely eliminate false positives (Strucken et al. 2016). In addition, parentage analysis based on SNPs has few predictable statistical problems that must be considered carefully and evaluated appropriately before substituting the classical STRs approach (Amorim \& Pereira 2005). For these reasons, microsatellites are preferred over SNPs for parentage testing.

Most of the microsatellite markers used for cattle identification and parentage verification are dimeric repeat motifs (Carolino et al. 2009; Stevanovic et al. 2010; van de Goor et al. 2009; Zhao 
277

278

279

280

281

282

283

284

285

286

287

288

289

290

291

292

293

294

295

296

297

298

299

300

301

302

303

et al. 2017). Similarly, all microsatellite markers used in the present study were dimeric. Repeat patterns have advantages as well as disadvantages. Dimeric microsatellites might have mutations or stutter bands that present allele interpretation errors (Walsh et al. 1996). On the contrary, during PCR conduction, trimeric, tetrameric, and pentameric repeat motifs demonstrated lower stutter slippage efficiency than dimeric microsatellites (Gill et al. 2005), and they ensured clear peak discrimination. On the other hand, an appropriate mutation rate might be beneficial for the verification of multigenerational parentage. This is because mutations might occur between an ancestor and a descendant's assumed father/mother, but not between an assumed father/mother and offspring, thus allowing the identification of true father/mother. For trimeric, tetrameric, pentameric, and hexameric microsatellites, gaps occurring during the sequence variant visualization within the repeat units can result in larger bin sizes than those of dimeric microsatellites (Gill et al. 2000). However, if the span of the sequence variants is too wide, it becomes increasingly difficult to confine the microsatellite markers within a fluorochrome to a single multiplex PCR system. Furthermore, multimeric repeats can also be compound. All of the microsatellite markers used in the present study were dimeric containing homogeneous repeat motifs, therefore, some markers can be labeled as one fluorochrome in a single multiplex PCR with unambiguous genotyping.

In order to use them in paternity testing and individual identification, microsatellite loci must have rare null alleles, and be at HWE, and the gametic association (linkage disequilibrium) should be absent. Null alleles are not amplified to detectable levels via PCR because of mutations at primer binding sites (Kline et al. 2011). The frequency of null alleles is mainly estimated by Mendelian incompatibilities (Strucken et al. 2016) and by comparing the observed and expected number of homozygotes at a locus (Dąbrowski et al. 2015). This fact must be considered when performing genotyping for parentage testing and when there is an apparent opposite homozygosity between parent and offspring. In the present study, the estimated null allele frequencies remained the highest for HEL10 $(\mathrm{F}(\mathrm{Null})=0.703)$ and MGTG4B $(\mathrm{F}(\mathrm{Null})=$ 0.163). Therefore, these loci were excluded from the core set used for parental identification. 
304

305

306

307

308

309

310

311

312

313

314

315

316

317

318

319

320

321

322

323

324

325

326

327

328

329

330

The formulae used to estimate the exclusion probabilities assume random mating, random association between alleles of different loci, and allele frequencies consistent with HWE. The 17 loci that were selected as core microsatellite markers were at HWE (Table 1), indicating that they can be used to calculate PE values.

The microsatellites used in cattle parentage testing could not be directly applied to yak identification due to uncertainty of whether the primers used for cattle would produce the desired results in yak. In the present study, the microsatellite primers were designed based on the yak gene sequences, and were then tested by PCR and electrophoresis. In addition, the allelic frequencies differed between the cattle and yak. Even among cattle breeds, the microsatellite PIC values differed (Mao et al. 2008), and the same might be applied to yak breeds (Zhang et al. 2008). Thus, while determining the PE values of parentage testing and individual identification for a new breed, allele frequencies and PIC values should be calculated de novo.

The microsatellite loci with PIC value $>0.5$ were selected for the identification panel. Nevertheless, the PIC values of these loci were still moderately lower than those used for cattle testing (Stevanovic et al. 2010; Vohra et al. 2017). Therefore, a high number of microsatellite markers were screened in the present study, and a set of 17 microsatellite markers proved to be sufficient for determining PE values. The combined exclusion probabilities of wrongly assigned sires were 99.718116\%, 99.997381\%, and 99.999998\% for PE1, PE2, and PEP, respectively. Similar results were reported in Angeln dairy cattle, with 16 microsatellites and approximately 99.9\% PE1 (Sanders et al. 2006). The PEP value calculated for the set of 17 microsatellites for parentage testing in the present study was $99.997381 \%$, which was marginally higher than that obtained for Swiss yaks (99.5\%) using 13 STR markers (Nguyen et al. 2005). Therefore, the set of microsatellite loci used in the present study significantly contributed to parental identification in the polled yak population.

We used several mapped cattle microsatellites to develop the sets of yak loci that were suitable for multiplex PCR amplification, and multiplex loading was conducted in a single run to reduce human errors, typing cost, and time. Nevertheless, selecting markers for a universal panel 
331 depends on the balance among the required panel accuracy, amplicon length, and ability to

332

333

334

335

336

337

338

339

340

341

342

343

344

345

346

347

348

349

350

351

352

353

354

355

356

357 undergo a successful multiplex reaction. Multiplex PCR amplifications are technically more difficult than their single-locus counterparts, but they are less likely to transfer across species than single-locus amplifications. Several multiplex PCR and loading optimization methods have been investigated for parentage testing of cattle. Nevertheless, these methods cannot be directly applied to yak identification. Therefore, we developed four multiplex amplifications (each containing four or five loci) and two multiplex loads (each containing eight or nine loci) running in two gel lanes. The main advantage of this system is that the allele length ranges do not overlap within the same fluorochrome.

Hence, we proposed that a combination of 17 microsatellites can yield a polled yak panel with enhanced processing efficiency, reliability, and utility. Moreover, this system uses the standard genotyping methods of DNA fragment analysis technology. Combined with likelihood-based parentage testing, these 17 markers will help improve breeding programs and accurately determine polled yak pedigrees. If this system is used to identify polled yaks that are not the descendants of the expected breeding male yak, breeders can eliminate them from the breeding group to ensure genetic purity and breed improvement. On the other hand, if the semen samples of high-grade male yaks are mixed with those of other males, the detection system can use DNA from semen and blood of the high-grade male yak for identification. Multiplex systems can also be used to rapidly assess the history, structure, and diversity of the breeding population, and these systems can reconstruct relationships among breeds. Furthermore, these multiplex systems might also be applied to other yak breeds with gene frequencies that are similar to that of the population tested in the present study.

Although the core set of microsatellite loci presented here was meaningful for yak parentage testing, this methodology still has the following limitations: (a) Since different yak breeds have different microsatellite genotypes, this core set was only suitable for parentage testing of polled yak; (b) The PIC values of the microsatellite loci are not sufficiently high to reduce the number of loci, thus avoiding low PE values; and (c) The number of panels for multiplex PCR reactions 
358

359

360

361

362

363

364

365

366

367

368

369

370

371

372

373

374

375

376

377

378 and multiplex loading still remained too high, leading to time-consuming and high cost. Therefore, a large number of microsatellites with high PIC values should be obtained for different yak breeds to develop efficient parentage test systems, with higher PE values and fewer markers. Furthermore, microsatellites should be suitable to each specific yak breed, and markers should be screened using single multiplex PCR reactions or multiplex loading.

\section{CONCLUSIONS}

A set of 17 microsatellite markers, which were assembled into four multiplex PCR reaction systems and genotyped in two multiplex loading systems, were identified and evaluated. The high variability displayed by these microsatellite loci demonstrated that highly precise genotyping panels might be used for individual genotyping, parentage verification, and individual identification. The microsatellites reported in this study could also be used to evaluate yak population structure, history, and diversity, which subsequently aids the genetic improvement of domestic yak.

\section{ACKNOWLEDGEMENTS}

We thank Hongli Jiang from Beijing UBioLab Genetics Technology Co., Ltd. for technical advice on genotype evaluation and multiplex system development. We also thank Editage for improving the language of this manuscript. We thank the Editor and the anonymous Reviewers for their constructive comments on the manuscript. 


\section{REFERENCES}

380

Amorim A, and Pereira L. 2005. Pros and cons in the use of SNPs in forensic kinship investigation: a comparative analysis with STRs. Forensic Sci Int 150:17-21. 10.1016/j.forsciint.2004.06.018

Barsila SR, Kreuzer M, Devkota NR, Ding L, and Marquardt S. 2014. Adaptation to Himalayan high altitude pasture sites by yaks and different types of hybrids of yaks with cattle. Livestock Science 169:125-136. 10.1016/j.livsci.2014.09.004

Carneiro Vieira ML, Santini L, Diniz AL, and Munhoz CdF. 2016. Microsatellite markers: what they mean and why they are so useful. Genetics and Molecular Biology 39:312-328. 10.1590/1678-4685-gmb-2016-0027

Carolino I, Sousa CO, Ferreira S, Carolino N, Silva FS, and Gama LT. 2009. Implementation of a parentage control system in Portuguese beef-cattle with a panel of microsatellite markers. Genetics and Molecular Biology 32:306-311. 10.1590/s1415-47572009005000026

Coetzer WG, Downs CT, Perrin MR, and Willows-Munro S. 2017. Testing of microsatellite multiplexes for individual identification of Cape Parrots (Poicephalus robustus): paternity testing and monitoring trade. Peerj 5. 10.7717/peerj.2900

Dąbrowski M, Bornelöv S, Kruczyk M, Baltzer N, and Komorowski J. 2015. 'True' null allele detection in microsatellite loci: a comparison of methods, assessment of difficulties and survey of possible improvements. Mol Ecol Resour 15:477-488.

Ding LM, Wang YP, Brosh A, Chen JQ, Gibb MJ, Shang ZH, Guo XS, Mi JD, Zhou JW, Wang HC, Qiu Q, and Long RJ. 2014. Seasonal heat production and energy balance of grazing yaks on the Qinghai-Tibetan plateau. Animal Feed Science and Technology 198:83-93. 10.1016/j.anifeedsci.2014.09.022

Eggen A. 2012. The development and application of genomic selection as a new breeding paradigm. Animal Frontiers 2:10-15. 10.2527/af.2011-0027

Estoup A, Jarne P, and Cornuet JM. 2002. Homoplasy and mutation model at microsatellite loci and their consequences for population genetics analysis. Molecular Ecology 11:1591-1604. 10.1046/j.1365294X.2002.01576.x

Gill P, Curran J, and Elliot K. 2005. A graphical simulation model of the entire DNA process associated with the analysis of short tandem repeat loci. Nucleic Acids Res 33:632-643. 10.1093/nar/gki205

Gill P, Whitaker J, Flaxman C, Brown N, and Buckleton J. 2000. An investigation of the rigor of interpretation rules for STRs derived from less than 100 pg of DNA. Forensic Sci Int 112:17-40. 10.1016/s0379-0738(00)001584

Hu Q, Ma T, Wang K, Xu T, Liu J, and Qiu Q. 2012. The Yak genome database: an integrative database for studying yak biology and high-altitude adaption. BMC Genomics 13:(7 November 2012)-(2017 November 2012).

Israel C, and Weller J. 2000. Effect of misidentification on genetic gain and estimation of breeding value in dairy cattle populations. J Dairy Sci 83:181-187.

Jan C, and Fumagalli L. 2016. Polymorphic DNA microsatellite markers for forensic individual identification and parentage analyses of seven threatened species of parrots (family Psittacidae). Peerj 4:e2416.

Jeong H, Choi B-H, Lee H-E, Gim J-A, and Kim H-S. 2015. Microsatellite analysis of genetic variation and structure in Korean and exotic dog breeds. Genes \& Genomics 37:819-827. 10.1007/s13258-015-0313-2

Kaiser S, Taylor S, Chen N, Sillett T, Bondra E, and Webster M. 2017. A comparative assessment of SNP and 
microsatellite markers for assigning parentage in a socially monogamous bird. Mol Ecol Resour 17:183-193.

Kalinowski ST, Taper ML, and Marshall TC. 2010. Revising how the computer program CERVUS accommodates genotyping error increases success in paternity assignment (vol 16, pg 1099, 2007). Molecular Ecology 19:1512-1512. 10.1111/j.1365-294X.2010.04544.x

Kang SW, Lee SY, Chio DH, Kang HJ, Hu MB, and Yang YJ. 2016. Statistical analysis of alleles in 4703 thoroughbred racing horses using fifteen microsatellite DNA markers. Journal of Animal Science 94:88-88. 10.2134/jas2016.94supplement488x

Kline MC, Hill CR, Decker AE, and Butler JM. 2011. STR sequence analysis for characterizing normal, variant, and null alleles. Forensic Science International-Genetics 5:329-332. 10.1016/j.fsigen.2010.09.005

Li D, Chai Z, Ji Q, Zhang C, Xin J, and Zhong J. 2013. Genetic diversity of DNA microsatellite for Tibetan yak. Hereditas (Beijing) 35:175-184.

Linacre A, Gusmao L, Hecht W, Hellmann AP, Mayr WR, Parson W, Prinz M, Schneider PM, and Morling N. 2011. ISFG: Recommendations regarding the use of non-human (animal) DNA in forensic genetic investigations. Forensic Science International-Genetics 5:501-505. 10.1016/j.fsigen.2010.10.017

Mao Y, Chang H, Yang Z, Zhang L, Xu M, Chang G, Sun W, Song G, and Ji D. 2008. The analysis of genetic diversity and differentiation of six Chinese cattle populations using microsatellite markers. J Genet Genomics 35:25-32.

Medhammar E, Wijesinha-Bettoni R, Stadlmayr B, Nilsson E, Charrondiere UR, and Burlingame B. 2012. Composition of milk from minor dairy animals and buffalo breeds: a biodiversity perspective. Journal of the Science of Food and Agriculture 92:445-474. 10.1002/jsfa.4690

Mizuno S, Ishizaki T, Toga H, Sakai A, Isakova J, Taalaibekova E, Baiserkeev Z, Kojonazarov B, and Aldashev A. 2015. Endogenous Asymmetric Dimethylarginine Pathway in High Altitude Adapted Yaks. Biomed Research International. 10.1155/2015/196904

Nguyen T, Genini S, Ménétrey F, Malek M, Vögeli P, Goe M, and Stranzinger G. 2005. Application of bovine microsatellite markers for genetic diversity analysis of Swiss yak (Poephagus grunniens). Anim Genet 36:484-489.

Qiu Q, Zhang G, Ma T, Qian W, Wang J, Ye Z, Cao C, Hu Q, Kim J, Larkin DM, Auvil L, Capitanu B, Ma J, Lewin HA, Qian $X$, Lang Y, Zhou R, Wang L, Wang K, Xia J, Liao S, Pan S, Lu X, Hou H, Wang Y, Zang X, Yin Y, Ma H, Zhang J, Wang Z, Zhang Y, Zhang D, Yonezawa $T$, Hasegawa M, Zhong $Y$, Liu W, Zhang Y, Huang Z, Zhang S, Long R, Yang H, Wang J, Lenstra JA, Cooper DN, Wu Y, Wang J, Shi P, and Liu J. 2012. The yak genome and adaptation to life at high altitude. Nature Genetics 44:946-+. 10.1038/ng.2343

Raymond M, and Rousset F. 1995. GENEPOP (VERSION-1.2) - POPULATION-GENETICS SOFTWARE FOR EXACT TESTS AND ECUMENICISM. Journal of Heredity 86:248-249.

Rohrer GA, Freking BA, and Nonneman D. 2007. Single nucleotide polymorphisms for pig identification and parentage exclusion. Animal Genetics 38:253-258. 10.1111/j.1365-2052.2007.01593.x

Rosa AJM, Sardina MT, Mastrangelo S, Tolone M, and Portolano B. 2013. Parentage verification of Valle del Belice dairy sheep using multiplex microsatellite panel. Small Ruminant Research 113:62-65. 10.1016/j.smallrumres.2013.03.021

Rousset F. 2008. GENEPOP ' 007: a complete re-implementation of the GENEPOP software for Windows and Linux. Molecular Ecology Resources 8:103-106. 10.1111/j.1471-8286.2007.01931.x

Sanders K, Bennewitz J, and Kalm E. 2006. Wrong and missing sire information affects genetic gain in the Angeln dairy cattle population. J Dairy Sci 89:315-321. 
Schnabel RD, Ward TJ, and Derr JN. 2000. Validation of 15 microsatellites for parentage testing in North American bison, Bison bison and domestic cattle. Animal Genetics 31:360-366. 10.1046/j.1365-2052.2000.00685.x

Sharma R, Kishore A, Mukesh M, Ahlawat S, Maitra A, Pandey A, and Tantia M. 2015. Genetic diversity and relationship of Indian cattle inferred from microsatellite and mitochondrial DNA markers. BMC Genet 16:73.

Slate J, Marshall T, and Pemberton J. 2000. A retrospective assessment of the accuracy of the paternity inference program CERVUS. Molecular Ecology 9:801-808. 10.1046/j.1365-294x.2000.00930.x

Stevanovic J, Stanimirovic Z, Dimitrijevic V, and Maletic M. 2010. Evaluation of 11 microsatellite loci for their use in paternity testing in Yugoslav Pied cattle (YU Simmental cattle). Czech Journal of Animal Science 55:221-226.

Strucken EM, Lee SH, Lee HK, Song KD, Gibson JP, and Gondro C. 2016. How many markers are enough? Factors influencing parentage testing in different livestock populations. Journal of Animal Breeding and Genetics 133:13-23. 10.1111/jbg.12179

van de Goor LHP, Panneman H, and van Haeringen WA. 2009. A proposal for standardization in forensic bovine DNA typing: allele nomenclature of 16 cattle-specific short tandem repeat loci. Animal Genetics 40:630-636. 10.1111/j.1365-2052.2009.01891.x

Vohra V, Sodhi M, Niranjan SK, Mishra AK, Chopra A, Kumar M, and Joshi BK. 2017. Characterization of rare migratory cattle and evaluation of its phylogeny using short-tandem-repeat-based markers. Journal of Applied Animal Research 45:355-363. 10.1080/09712119.2016.1194843

Walsh PS, Fildes NJ, and Reynolds R. 1996. Sequence analysis and characterization of stutter products at the tetranucleotide repeat locus vWA. Nucleic Acids Res 24:2807-2812. 10.1093/nar/24.14.2807

Wang G, Chen S, Chao T, Ji Z, Hou L, Qin Z, and Wang J. 2017. Analysis of genetic diversity of Chinese dairy goats via microsatellite markers. J Anim Sci 95:2304-2313.

Wang L-L, Yu Q-L, Han L, Ma X-L, Song R-D, Zhao S-N, and Zhang W-H. 2018. Study on the effect of reactive oxygen species-mediated oxidative stress on the activation of mitochondrial apoptosis and the tenderness of yak meat. Food Chemistry 244:394-402. 10.1016/j.foodchem.2017.10.034

Weising K, Winter P, Huttel B, and Kahl G. 1998. Microsatellite markers for molecular breeding. Journal of Crop Production 1:113-143.

Wu J. 2016. The distributions of Chinese yak breeds in response to climate change over the past 50 years. Animal Science Journal 87:947-958. 10.1111/asj.12526

Zhang G, Chen W, Xue M, Wang Z, Chang H, Han X, Liao X, and Wang D. 2008. Analysis of genetic diversity and population structure of Chinese yak breeds (Bos grunniens) using microsatellite markers. J Genet Genomics 35:233-238.

Zhao J, Zhu C, Xu Z, Jiang X, Yang S, and Chen A. 2017. Microsatellite markers for animal identification and meat traceability of six beef cattle breeds in the Chinese market. Food Control 78:469-475. 10.1016/j.foodcont.2017.03.017 


\section{Table 1 (on next page)}

Genetic information for the 35 polymorphic microsatellite loci. 
1 Table 1 Genetic information for the 35 polymorphic microsatellite loci.

\begin{tabular}{|c|c|c|c|c|c|c|c|c|}
\hline Locus ID & Range (bp) & $\begin{array}{l}\text { Repeat } \\
\text { motif }\end{array}$ & $\mathrm{N}_{\mathrm{A}}$ & $\mathrm{H}_{\mathrm{O}}$ & $\mathrm{H}_{\mathrm{E}}$ & PIC & HWE & $\begin{array}{l}\text { F } \\
\text { (Null) }\end{array}$ \\
\hline BM720 & $160-168$ & $\mathrm{AC}$ & 5 & 0.574 & 0.588 & 0.541 & NS & 0.0127 \\
\hline BM1818 & $239-251$ & $\mathrm{AC}$ & 5 & 0.325 & 0.329 & 0.302 & NS & 0.0031 \\
\hline BM2113 & $115-133$ & TG & 8 & 0.673 & 0.662 & 0.623 & NS & 0.0067 \\
\hline BM2943 & 253 & $\mathrm{AC}$ & 1 & 0 & 0 & 0 & ND & ND \\
\hline CSSM013 & $156-160$ & TG & 3 & 0.294 & 0.309 & 0.272 & NS & 0.0180 \\
\hline CSSM029 & $182-194$ & $\mathrm{AC}$ & 6 & 0.517 & 0.466 & 0.417 & NS & 0.0623 \\
\hline CSSM033 & $309-317$ & TG & 3 & 0.137 & 0.130 & 0.126 & ND & 0.0261 \\
\hline CSSM036 & $157-179$ & TG & 10 & 0.677 & 0.693 & 0.646 & NS & 0.0116 \\
\hline CSSM041 & $128-134$ & TG & 4 & 0.457 & 0.428 & 0.402 & NS & 0.0427 \\
\hline CSSME 070 & $249-259$ & $\mathrm{AC}$ & 5 & 0.513 & 0.526 & 0.412 & NS & 0.0093 \\
\hline HAUT24 & $217-231$ & $\mathrm{AC}$ & 8 & 0.628 & 0.633 & 0.572 & NS & 0.0059 \\
\hline HEL5 & $214-228$ & TG & 6 & 0.182 & 0.181 & 0.174 & ND & 0.0055 \\
\hline HEL6 & $247-265$ & GT & 8 & 0.686 & 0.754 & 0.721 & NS & 0.0427 \\
\hline HEL10 & 171-177 & TG & 4 & 0.091 & 0.502 & 0.417 & $* * *$ & 0.7025 \\
\hline ILSTS006 & $162-170$ & GT & 4 & 0.453 & 0.448 & 0.374 & NS & 0.0095 \\
\hline ILSTS008 & $172-184$ & $\mathrm{AC}$ & 6 & 0.577 & 0.550 & 0.446 & NS & 0.0257 \\
\hline ILSTS028 & $261-293$ & GT & 11 & 0.806 & 0.838 & 0.815 & NS & 0.0194 \\
\hline ILSTS030 & $150-156$ & GT & 4 & 0.106 & 0.129 & 0.123 & ND & 0.1033 \\
\hline INRA005 & 186-202 & $\mathrm{CA}$ & 5 & 0.607 & 0.604 & 0.529 & NS & 0.0058 \\
\hline INRA023 & 184-204 & TG & 10 & 0.753 & 0.780 & 0.752 & NS & 0.0162 \\
\hline INRA035 & 104 & TG & 1 & 0 & 0 & 0 & ND & ND \\
\hline INRA037 & $296-312$ & $\mathrm{CA}$ & 8 & 0.688 & 0.770 & 0.730 & NS & 0.0576 \\
\hline INRA063 & $175-187$ & TG & 7 & 0.604 & 0.642 & 0.585 & NS & 0.0317 \\
\hline MGTG4B & $245-255$ & $\mathrm{AC}$ & 5 & 0.196 & 0.273 & 0.254 & ** & 0.1631 \\
\hline MM12 & $109-121$ & GT & 4 & 0.085 & 0.087 & 0.084 & ND & 0.0242 \\
\hline РОТСНА & $128-148$ & $\mathrm{CA}$ & 8 & 0.573 & 0.585 & 0.540 & NS & 0.0227 \\
\hline RM099 & 233 & $\mathrm{CA}$ & 1 & 0 & 0 & 0 & ND & ND \\
\hline SPS115 & $231-261$ & $\mathrm{CA}$ & 12 & 0.782 & 0.806 & 0.780 & NS & 0.0146 \\
\hline TGLA57 & $253-263$ & GT & 6 & 0.348 & 0.340 & 0.322 & NS & 0.0120 \\
\hline TGLA126 & 209-223 & TG & 8 & 0.587 & 0.690 & 0.639 & NS & 0.0819 \\
\hline TGLA227 & $210-222$ & $\mathrm{AC}$ & 5 & 0.611 & 0.617 & 0.538 & NS & 0.0015 \\
\hline YAK07 & 323-339 & TG & 9 & 0.63 & 0.621 & 0.589 & NS & 0.0104 \\
\hline YAK08 & $321-343$ & $\mathrm{CA}$ & 8 & 0.698 & 0.676 & 0.612 & NS & 0.0218 \\
\hline YAK11 & $306-314$ & GT & 7 & 0.679 & 0.666 & 0.597 & NS & 0.0096 \\
\hline YAK12 & 259-279 & GT & 8 & 0.549 & 0.522 & 0.406 & NS & 0.0269 \\
\hline
\end{tabular}

24 Note:

25 Font bold, core microsatellite loci are indicated in bold; Range, range of allele sizes; Repeat motif, repeat motif of

26 microsatellites; $\mathrm{N}_{\mathrm{A}}$, number of alleles found; $\mathrm{H}_{\mathrm{O}}$, observed heterozygosity; $\mathrm{H}_{\mathrm{E}}$, expected heterozygosity; PIC,

27 polymorphism information content; HWE, departure from Hardy-Weinberg equilibrium; NS, not significant; ND,

28 not done; ${ }^{* *}$, significant at the $1 \%$ level; ${ }^{* *}$, significant at the $0.1 \%$ level; F (Null), estimated null allele frequency. 


\section{Table 2 (on next page)}

Detailed primer information for the core microsatellite loci identified in the present study. 
1 Table 2 Detailed primer information for the core microsatellite loci identified in the present study.

2

3

4

5

6

7

8

9

10

11

12

13

14

15

16

17

18

19

20

21

22

23

24

25

26

27

28

\section{Table 2 Detailed primer information for the core mierosatelite loci identified in the present study.}

\begin{tabular}{|c|c|c|c|c|c|c|c|c|c|c|c|c|c|c|}
\hline $\begin{array}{l}\text { Locus } \\
\text { name }\end{array}$ & Forward primer $\left(5^{\prime} \rightarrow 3^{\prime}\right)$ & Reverse primer $\left(5^{\prime} \rightarrow 3^{\prime}\right)$ & $\begin{array}{l}\mathrm{C} \\
{[\mathrm{mM}]}\end{array}$ & $\begin{array}{l}\mathrm{Ta} \\
\left.{ }^{\circ} \mathrm{C}\right)\end{array}$ & PMP & PML & Fluoro & RM & EP1 & EP2 & EPP & EI & ESI & $\mathrm{CL}$ \\
\hline BM720 & GAAATCAACAAGTTCCAATCCTG & ATCTCATTCTTGTGTCATGGATGA & 3.5 & 56 & 3 & 2 & 6-FAM & (AC) & 0.189 & 0.353 & 0.532 & 0.783 & 0.489 & 13 \\
\hline BM2113 & GCTGCCTTCTACCAAATACCC & CTTCCTGAGAGAAGCAACACC & 1.9 & 55 & 1 & 1 & 6-FAM & (TG) & 0.261 & 0.439 & 0.636 & 0.847 & 0.542 & 2 \\
\hline CSSM036 & GATAACTCAACCACACGTCT & AAGAAGTACTGGTTGCCAATCG & 2.8 & 56 & 2 & 1 & 6-FAM & (TG) & 0.283 & 0.456 & 0.644 & 0.859 & 0.561 & 27 \\
\hline HAUT24 & CTCTGCCTTTGTCCCTGTCT & CCAAACCCCCTACCCACA & 5.7 & 56 & 2 & 1 & TAMARA & $(\mathrm{AC})$ & 0.221 & 0.377 & 0.552 & 0.805 & 0.517 & 22 \\
\hline HEL6 & GGACACGACTGAGCAAGTAACA & GCTTTGGCAGGCAGATACAT & 4.0 & 56 & 1 & 1 & HEX & (GT) & 0.367 & 0.549 & 0.743 & 0.907 & 0.603 & 1 \\
\hline ILSTS028 & AGAAGAGTGTACCTCCTCCCAC & TCCAGATTTTGTACCAGACCAT & 4.6 & 56 & 1 & 1 & TAMARA & (GT) & 0.503 & 0.673 & 0.847 & 0.953 & 0.656 & 11 \\
\hline INRA005 & CTTCAGGCATACCCTACACCA & GGGGAATCTGTGGAGGAGTT & 8.3 & 56 & 4 & 2 & 6-FAM & (CA) & 0.190 & 0.328 & 0.483 & 0.768 & 0.494 & 12 \\
\hline INRA023 & ATTTCCCTTCTGACTGGTACTTC & GTGTCCCTCCTCTAATCCCTAA & 3.0 & 55 & 3 & 2 & HEX & (TG) & 0.408 & 0.589 & 0.782 & 0.924 & 0.620 & 3 \\
\hline INRA037 & GCTACAATCCAGACTGAGCACG & GACACGGCTTAGCGACTGAA & 3.1 & 57 & 3 & 2 & HEX & (CA) & 0.370 & 0.549 & 0.731 & 0.909 & 0.611 & 10 \\
\hline INRA063 & AAACCACAGAAATGCTTGGAAG & ATTTGCACAAGCTAAATCTAACAA & 3.6 & 56 & 1 & 1 & TAMARA & (TG) & 0.228 & 0.390 & 0.566 & 0.816 & 0.524 & 18 \\
\hline РОТСНА & ATGCCAACTTTTCCCATCACT & GTAAACACAGTTCCCTGGAGAGA & 3.5 & 56 & 2 & 1 & HEX & (CA) & 0.192 & 0.357 & 0.540 & 0.783 & 0.488 & 15 \\
\hline SPS115 & AAAGTGACACAACAGCTTCACC & ACCGAGTGTCCTAGTTTGGC & 4.6 & 55 & 4 & 2 & TAMARA & (CA) & 0.452 & 0.628 & 0.814 & 0.938 & 0.636 & 15 \\
\hline TGLA126 & ATGAGAGAGGCTTCTGGGATG & CTTCACCATTGGACCACGAG & 3.7 & 56 & 4 & 2 & HEX & (TG) & 0.272 & 0.444 & 0.625 & 0.854 & 0.558 & 20 \\
\hline TGLA227 & CAAAGGAGCATAACTTTACAGCA & AGCCCTAACCATTGGACAGC & 4.9 & 57 & 3 & 2 & TAMARA & (AC) & 0.200 & 0.337 & 0.494 & 0.775 & 0.501 & 18 \\
\hline YAK07 & TAACAAAGCTGCTGGGAACAT & CGGAGTCACTTTCCTCACCTAT & 2.4 & 56 & 4 & 2 & HEX & (TG) & 0.230 & 0.412 & 0.615 & 0.825 & 0.516 & 1 \\
\hline YAK08 & ACTGGAGTAGGTTGCCCTGC & CCTGGCTTGGTCCTGTCTCT & 3.8 & 56 & 2 & 1 & HEX & (CA) & 0.247 & 0.405 & 0.572 & 0.831 & 0.545 & 6 \\
\hline YAK11 & TCCCCTCACTCCTCATTGGT & TGCAGGCAGTTTCTTACCAGT & 4.4 & 56 & 3 & 2 & TAMARA & (GT) & 0.233 & 0.387 & 0.548 & 0.820 & 0.537 & 1 \\
\hline Combined & & & & & & & & & $\begin{array}{l}0.9971 \\
8116\end{array}$ & $\begin{array}{l}0.9999 \\
7381\end{array}$ & $\begin{array}{l}0.9999 \\
9998\end{array}$ & $\begin{array}{l}>0.999 \\
99999\end{array}$ & $\begin{array}{l}0.9999 \\
9899\end{array}$ & \\
\hline
\end{tabular}

Note:

C, concentration of primers; Ta, annealing temperature; PMP, panel numbers for multiplex PCR; PML, panel numbers for multiplex loading; Fluoro, fluorescent dye; RM, repeat motif of microsatellites; PE1, the average probability that the set of loci will exclude an unrelated candidate parent from parentage of an arbitrary offspring when the genotype of the other parent is unknown; PE2, the average probability that the set of loci will exclude an unrelated candidate parent from parentage of an arbitrary offspring when the genotype of the other parent is known; PEP, the average probability that the set of loci will exclude a pair of unrelated candidate parents from parentage of an arbitrary offspring; PEI, the average probability that the set of loci will differentiate between two randomlyselected individuals; PESI, the average probability that the set of loci will differentiate between two randomly-selected full siblings; CL, locations of microsatellite loci on cattle chromosoms. 


\section{Figure 1}

Typical fluorescence signal s of detections for the core 17 microsatellite loci.

A: Panel 1, B: Panel 2, C: Panel 3, D: Panel 4. The "Panels" stand for the multiplex PCR

groups. The colors of fluorochrome 6-FAM, HEX, and TAMARA are showed by blue, green, and black respectively. The numbers above the sharp peaks represent length scales based on the internal size standard (bp). The numbers below the sharp peaks represent fragment lengths of PCR amplifications (bp). 
A
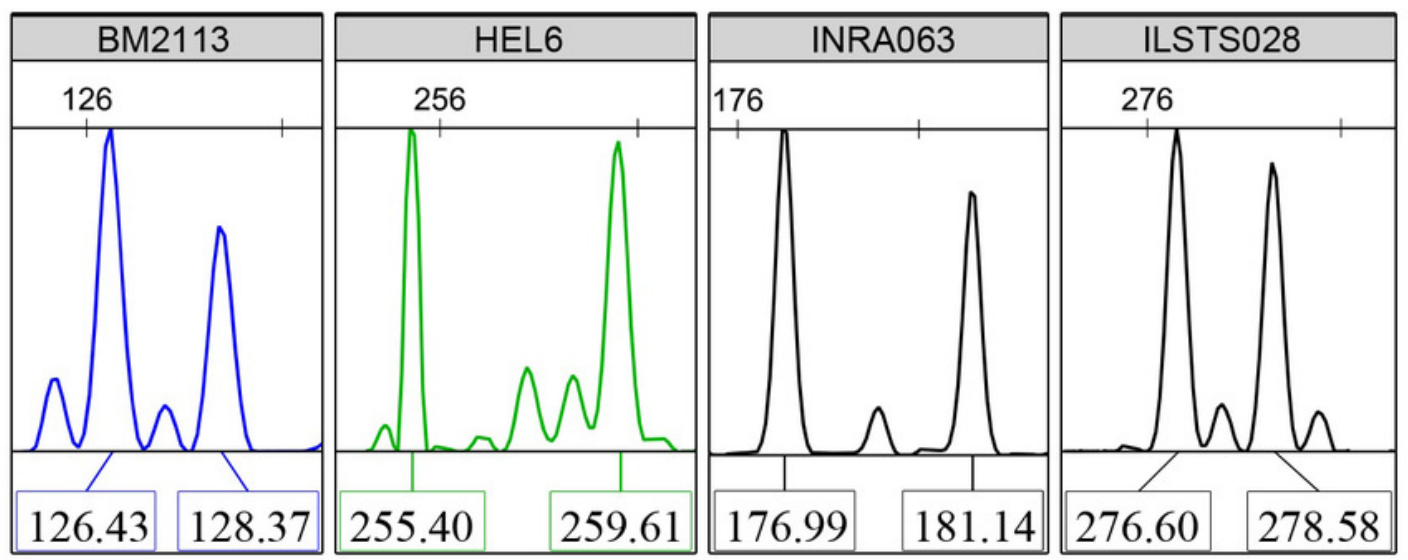

B
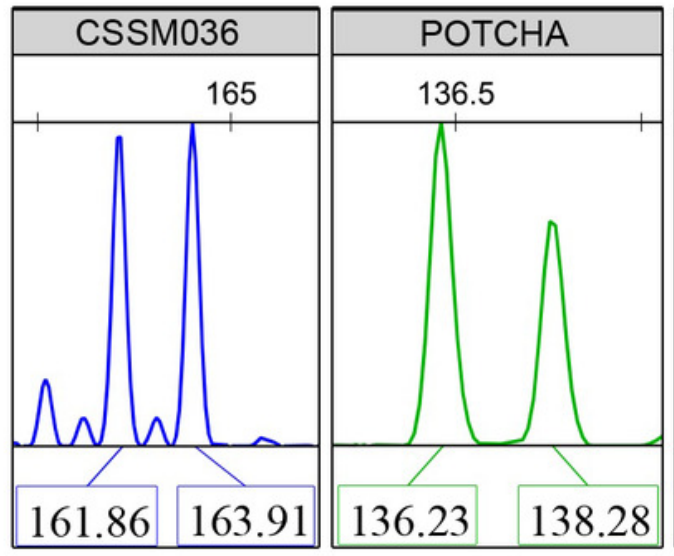

C
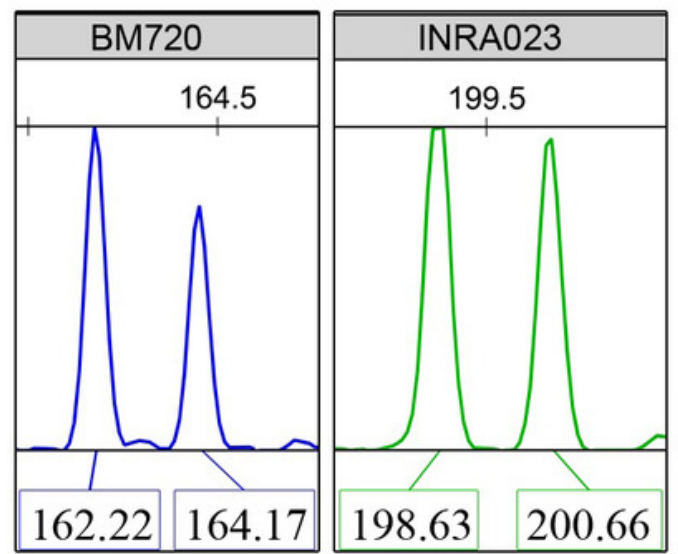

D
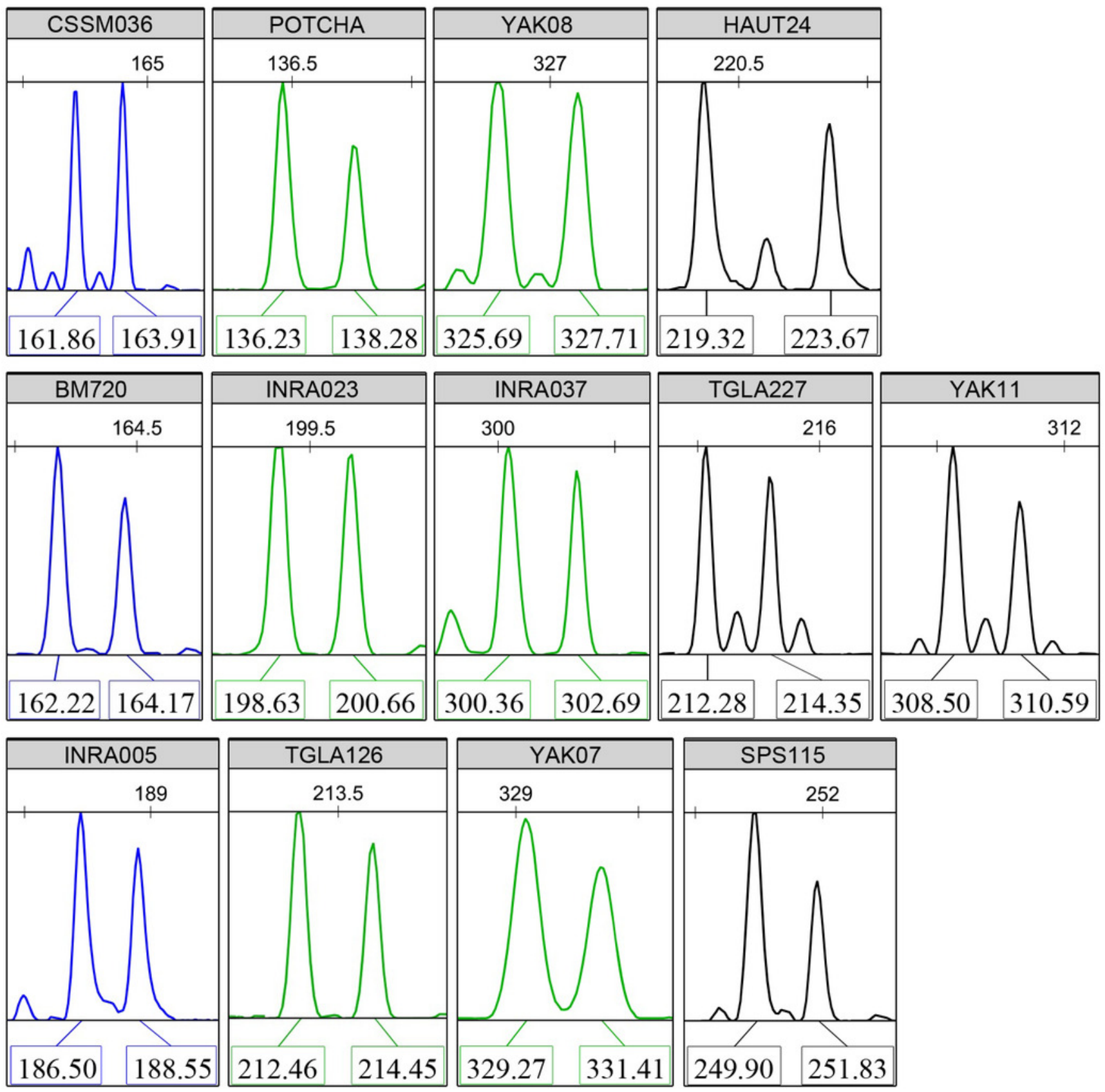